Trauma

\title{
Developing a Clinical Guideline for CT Scans in Closed Head Injury
}

\author{
Chengyuan $\mathrm{Wu}, \mathrm{MD}$, Jack Jallo, MD \\ Department of Neurological Surgery, Thomas Jefferson University, Philadelphia, PA
}

Several years ago, every patient that was brought to Thomas Jefferson University Hospital with a closed head injury would receive a CT scan - regardless of whether or not that patient already had a scan performed at an outside institution. If the scan demonstrated any intracranial pathology, then a repeat CT scan was performed 12 hours after the initial scan as long as the patient remained neurologically stable. Ultimately, every patient received two CT scans at our institution separated by 12 hours.

Now, each patient is followed by a total of three CT scans. If a patient had undergone a CT scan at an outside institution, this scan was loaded onto the Jefferson system and was considered their first CT scan. A follow-up CT scan was to be performed 6 hours after the initial scan if any intracranial pathology was noted. As long as the patient remained neurologically stable, a third and final CT scan was to be performed sometime between 12 to 24 hours after the initial scan. Therefore, patients with non-operative intracranial hemorrhage received two or three CT scans at our institution and were observed for at least 24 hours.

In both situations, routine follow-up imaging was performed on all patients with an initial intracranial lesion resulting from head trauma. This practice is founded on the recommendation that "early imaging, rather than awaiting neurological deterioration, reduces the delay in detection and treatment of acute intracranial injury." This is based largely on the existing literature regarding epidural hematomas (EDH) and severe head injury. More than $50 \%$ of patients with severe head injuries have progression of findings on CT scan that otherwise would go undetected due to their poor initial clinical status. ${ }^{1}$ Case reports have illustrated the need for repeat imaging in the setting of "ultra-early" CT scans, which may not capture a developing hematoma. ${ }^{2}$ In addition, EDHs have been reported to be more likely to enlarge if captured within 6 hours of injury ${ }^{3}$ - again enforcing the importance of repeat imaging for these patients. At the same time, while such case reports and small case series have demonstrated the importance of imaging at least 6 hours after the initial injury, patients who have already had a negative CT scan generally do not get a repeated scan, - even if the negative scan was performed within 6 hours of their injury.

Overall, this protocol is based on such literature and the concern for progression of intracranial lesions within the first 24 hours after a traumatic event - and the importance of changes at the 6 hour mark. The low threshold for performing an initial and subsequent follow-up CT scans allows for a high sensitivity, as is desired in an effective screening tool. Other factors that need to be considered, however, are the effects of radiation exposure and the increased costs of serial imaging. With regards to the former, the Scottish Intercollegiate Guidelines Network determined that the risk of developing a fatal cancer from a single CT scan of the head is approximately 1 in 10,000. There is little literature regarding the determination of the cost-effectiveness of routine serial CT scanning. A recent article in the Journal of Trauma, however, illustrated the slight increase in cost-effectiveness in routine

\section{Table 1. Previously Published Guidelines Relevant to Head Trauma}

\begin{tabular}{|l|l|}
\hline Scottish Intercollegiate Guidelines Network (SIGN) & May 2009 \\
\hline Veterans Association/Department of Defense & April 2009 \\
\hline American College of Emergency Physicians & August 2008 \\
\hline American College of Radiologists & 2008 \\
\hline National Institute of Health & September 2007 \\
\hline Brain Trauma Foundation & March 2006 \\
\hline
\end{tabular}

serial scanning. "Since delayed hematomas are uncommon and expectant management is usually successful, relatively few patients benefit from routine CT scanning. However, the consequences of missing a delayed hematoma until permanent brain damage has occurred is so great, that the reported values of these parameters favors routine repeat scanning. ${ }^{4}$ Contrary to this, however, some insurance companies have recently ceased reimbursements for routine serial imaging. ${ }^{5}$

Given the variation in clinical practice regarding imaging protocols of patients suffering from head trauma, it would be useful to develop a guideline to help create some form of consensus regarding the standard of care. This may also help maintain appropriate healthcare coverage for this patient population.

\section{Researching Clinical \\ Recommendations}

The US Department of Health and Human Services website (www.guidelines.gov) was searched for the term "head trauma." The following guidelines are applicable to management of patients with head trauma (Table 1).

Each of these guidelines included recommendations regarding diagnosis of intracranial pathology using CT scan. After reviewing these guidelines, it was found that there was a great deal of similarity between the guidelines released by SIGN and those released since the guidelines released by the Brain Trauma Foundation in 2006. Most of these guidelines delineated the clinical criteria for selecting patients who should undergo an initial CT scan; and did not clearly outline who should undergo repeat or serial imaging. The guidelines set forth by the Brain Trauma Foundation, however, were specific to patients with intracranial pathology and outlined not only surgical, but also nonsurgical management of such patients. This included recommendations regarding serial imaging using CT scan. Therefore, it would be most useful to specifically analyze and compare the SIGN guidelines and the Brain Trauma Foundation guidelines.

The SIGN guidelines were robustly created by a systematic review of current literature on early management of head trauma. This was followed 


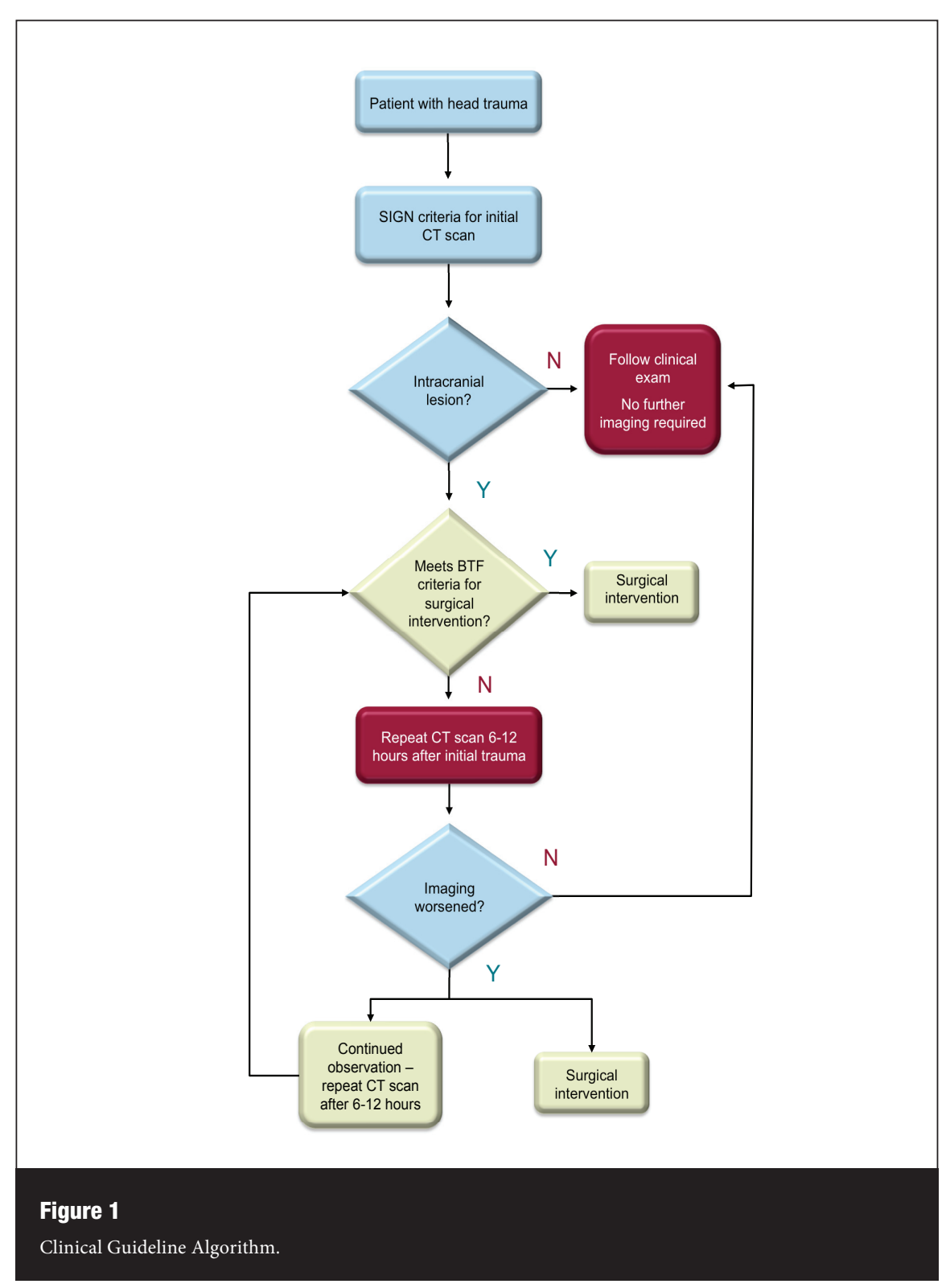

by a peer review process of the recommendations generated. It is the same methodology that this organization has used for a wide array of guidelines. The population included in the formulation of this guideline was quite broad - including all patients of any age with any degree of head trauma. It is therefore extremely applicable to our patient population - since it does not specifically exclude any particular cohort. At the same time, because of the scope of this document, there is a significant amount that does not directly address the clinical practice variation at hand. One section does focus in order to determine their validity. Ultimately, this analysis demonstrated high sensitivity and low specificity for both studies. "The estimated potential reduction in CT scans for patients with minor head injury would be $3.0 \%$ for the adapted NOC and $37.3 \%$ for the adapted CCHR." The major limitation of this analysis was that the inclusion criteria were not strictly followed in that not all of the patients included had loss of consciousness. At the same time, this apparent flaw also makes the results more readily generalized. Although there were few other exclusion criteria, the fact that only mild head trauma was included does limit the generality of these findings. At the same time, since these guidelines intend to provide guidance in situations when obtaining a CT scan is questionable, it does not necessarily need to include moderate or severe head trauma - situations when a CT scan should most certainly be performed. Overall, this study does indeed bring validity to the CCHR and NOC, which in turn strengthens the guidelines set forth by SIGN.

The Brain Trauma Foundation guidelines were also generated by a systematic review of current literature and followed by a peer review process of the recommendations generated. Unlike the SIGN guidelines, the BTF guidelines are targeted only towards the management of patients with post-traumatic intracranial mass lesions within the first 10 days of the trauma. While this population is somewhat more limited than the SIGN population, it still encompasses the population of patients we see at our institution. In fact, it is more tailored to our needs as Neurosurgical consults are typically only called when there is an intracranial mass lesion. Since this cohort will by definition have undergone a CT scan to detect an intracranial lesion, the BTF guidelines do not outline who should undergo an initial CT scan. Instead, these guidelines delineate who should undergo surgical intervention and who can be followed clinically with serial CT scans.

In patients with acute epidural hematomas that are to be managed non-operatively, "the first follow-up CT scan in nonoperative patients should be obtained within 6 to 8 hours after TBI." In the studies reviewed regarding nonoperative management of $\mathrm{EDH}$, the percentage of patients that later required surgical intervention ranged from 6-19\% in groups of approximately 80 patients. Patient outcome was found to be highly dependent on the time between the onset of neurologic deterioration (anisocoria, coma, etc.) and surgical decompression, with better outcomes in patients who were treated within 1-2 hours. While the guidelines regarding sub- 
dural hematomas do not outline any protocols for serial imaging of non-operative patients, they again note that patient outcomes are significantly improved if surgical intervention occurs within 1-2 hours of neurologic deterioration.

The phenomenon of Delayed Traumatic Intracerebral Hematoma (DTICH) is a major reason why clinicians continue to perform routine serial CT scans. "The incidence of DTICH ranges from 3.3 to $7.4 \%$ in patients with moderate-to-severe TBI. Evacuated DTICH represent approximately $1.6 \%$ of all evacuated traumatic ICH and mortality ranges from 16 to $72 \%$. Therefore, the importance of careful monitoring and of serial CT scanning cannot be overemphasized." At the same time, such monitoring is only recommended for patients with an abnormal initial CT scan, as "the majority of studies show that all patients who develop clinically relevant DTICH have abnormal initial CT scans." The time course of observation recommended is 72 hours, as no patients with DTICH at this time required any surgical intervention; and mortality was noted only when this entity occurred within the first 48 hours from the time of injury.

One major limitation of these guidelines is the strength of data available. They noted that "there are no controlled clinical trials in the literature to support different forms of surgical management, or to support surgical versus conservative therapy." This unfortunately leads to the dilemma of 'garbage-in garbage-out'. In addition, as mentioned before, the population involved also excludes patients without an intracranial lesion and therefore, generalizations cannot be made regarding serial imaging of this cohort.

The BTF guidelines referenced a case series published in 1995, of 37 patients with severe head trauma $(\mathrm{GCS}<8)$ who demonstrated changes in follow-up CT scans performed between 2 and 12 hours after their initial injury. ${ }^{7}$ These 37 patients represented $9 \%$ of all severe head injury patients. Of these patients, only 22 patients (5\%) had lesions that worsened from their initial scan. "The median time from admission to the second CT examination was 9 hours in patients harboring lesions that evolved toward reabsorption, 6 hours in patients who developed their first surgical lesions." Once again, the importance of the 6 hour mark is referenced in terms of re-imaging. The important point made by this series is that important changes in intracranial lesions can occur without changes in clinical presentation in patients with severe head trauma. While this demonstrates the importance of serial imaging, it applies only to patients with severe head trauma and a GCS $<8$. Its findings cannot be generalized to all patients who have experienced trauma and most certainly does not apply to patients with mild injury or those who are neurologically intact. Moreover, as a relatively small case series, the strength of this evidence is simply Level II-2.

Both of these guidelines provide recommendations regarding CT imaging in patients suffering from head trauma. While the SIGN guidelines indicate which patients require an initial CT scan, the BTF guidelines indicate the importance of follow-up imaging in patients with a known intracranial lesion and poor neurologic exam or neurologic deterioration. The SIGN criteria for imaging appear more stringent in terms of who undergoes an initial CT scan in comparison with our institution. Similarly, the BTF guidelines recommend repeat imaging with CT scan only in patients with a poor neurologic examination at time of admission; whereas all patients with an intracranial lesion undergo repeat imaging at our institution, regardless of their clinical exam. The SIGN guidelines provide no specific information regarding repeat imaging. Overall, patients at our institution undergo more liberal imaging than that recommended by current treatment guidelines.

\section{Developing a Clinical Guideline}

In assimilating the recommendations of the guidelines reviewed, we can generate a specific guideline that is more applicable to our patient population. (Figure 1) This guideline is targeted towards physician evaluation and management of patients who have been subject to traumatic brain injury. Based on the guidelines delineated by SIGN, it dictates which patients should receive a CT scan after sustaining a closed head injury. It then also outlines a protocol of the timing of follow-up CT scan in patients who have been found to have an intracranial lesion. It is intended to be applied to any adult ( $\geq 18$ years old) who has sustained a closed head injury, since this is the common patient population studied in the guidelines reviewed. As with the BTF guidelines, the main limitation of this guideline is the lack of clinical data to determine a standard of care with regards to nonsurgical management of TBI patients.

All patients who have sustained head trauma undergo an initial evaluation in which the decision to perform a CT scan is made by applying the guidelines set forth by the Scottish Intercollegiate Guideline Network (SIGN).
If no intracranial lesion is seen on this initial CT head, then no further imaging is required unless there is a change in the patient's clinical exam. Although initial imaging should ideally occur after 6 hours of the injury in order to avoid missing lesions from ultra-early imaging, clinically significant lesions are rare and have only been published as case reports. ${ }^{8}$ In most situations, it is appropriate to simply follow the patient's clinical exam, rather than schedule repeat imaging.

There are a few important points to note when considering observation alone. First of all, the timing of serial imaging has not been found to correlate with patient outcomes. Instead, the timing of surgical intervention relative to neurologic decline has been the primary factor in determining patient outcome. This also follows that a worsening in follow-up CT scan does not necessarily require surgical intervention nor has it been shown to affect patient outcomes. For this reason, one can understand how it may be more appropriate to follow patients clinically as long as frequent neurologic checks are performed and an operating room is readily available at all times in the event of a neurologic decline. Such a scenario is more common in tertiary care centers, in which it may be more appropriate for patients to be observed for changes in their neurologic exam without routine scheduled CT scan. At the same time, if frequent neurologic exams cannot be performed or a patient cannot be rapidly brought to an operating room shortly after a change in his or her exam, it is more reasonable to schedule routine follow-up CT scans.

If the first CT scan performed demonstrates an intracranial lesion, then we can then apply the Brain Tumor Foundation criteria for surgical intervention of $\mathrm{EDH}, \mathrm{SDH}, \mathrm{IPH}$, and posterior fossa lesions as indicated here:

If no surgical intervention is indicated, repeat imaging should be performed between 6-12 hours after the initial trauma - regardless of the patient's clinical exam. "In the largest review to date, the current study found that $22 \%$ of the first SRBCT will show radiographic worsening of intracranial injury." ${ }^{\text {" }}$ While more studies have focused on radiographic changes in moderate and severe brain injury, recent literature as also shown that $8.7 \%$ of patients with an intracranial lesion and mild TBI have and evolution in their lesion and consequently require surgical intervention as well - and 2.4\% of the entire cohort had no neurologic deterioration. Whereas in patients with a GCS $\leq 8$, a total of $10.6 \%$ required surgical intervention after a subsequent CT scan, of which $1 \%$ of the entire 
cohort had no neurologic changes. ${ }^{10}$ These numbers are fairly comparable and support routine serial CT scans in all groups of patients with TBI - regardless of GCS. Another recent study outlined a simplified model of comparing the strategy of routine scanning to observation for all degrees of head trauma; and has shown that the former costs $\$ 12,670 /$ QALY. $^{11}$ Of course, imaging may also be required if there is a deterioration in the patient's neurologic exam.

If on repeat imaging, the intracranial lesion is stable or improved, no further imaging is required and the patient's clinical exam can be followed. If there is deterioration in the imaging findings, once again, the BTF criteria for surgical intervention should once again be applied. Once a CT scan is stable on two sequential studies, no further imaging is required and the patient's clinical exam can be followed.

\section{References}

1. Lobato RD, Alen JF, Perez-Nuñez A, Alday R, Gómez PA, Pascual B, Lagares A, Miranda P, Arrese I, Kaen A. Value of serial CT scanning and intracranial pressure monitoring for detecting new intracranial mass effect in severe head injury patients showing lesions type I-II in the initial CT scan. Neurocirugia. 2005 Jun;16(3):217-34.

2. Smith HK, Miller JD. The danger of an ultra-early computed tomographic scan in a patient with an evolving acute epidural hematoma. Neurosurgery. 1991 Aug;29(2):258-60.

3. Knuckey NW, Gelbard S, Epstein MH. The management of "asymptomatic" epidural hematomas. A prospective study. J Neurosurg. 1989 Mar;70(3):392-6.

4. Stein SC, Fabbri A, Servadei F. Routine serial computed tomographic scans in mild traumatic brain injury: when are they cost-effective? J Trauma. 2008 Jul;65(1):66-72.

5. www.anthem.com/provider/noapplication/ $\mathrm{f} 1 / \mathrm{s} 0 / \mathrm{t} 0 /$ pw_b144255.pdf

6. Smits M, Dippel DW, de Haan GG, Dekker HM, Vos PE, Kool DR, Nederkoorn PJ, Hofman PA, Twijnstra A, Tanghe HL, Hunink MG. External validation of the Canadian CT Head Rule and the New Orleans Criteria for CT scanning in patients with minor head injury. JAMA. $2005 \mathrm{Sep}$ 28;294(12):1519-25.

7. Servadei F, Nanni A, Nasi M, Zappi D, Vergoni G, Giuliani G, Arista A. Evolving brain lesions in the first 12 hours after head injury: Analysis of 37 comatose patients. Neurosurgery 37:899-906, 1995.

8. Smith HK, Miller JD. The danger of an ultra-early computed tomographic scan in a patient with an evolving acute epidural hematoma. Neurosurgery. 1991 Aug;29(2):258-60.

9. Thomas BW, Mejia VA, Maxwell RA, Dart BW, Smith PW, Gallagher MR, Claar SC, Greer SH, Barker DE. Scheduled repeat $\mathrm{CT}$ scanning for traumatic brain injury remains important in assessing head injury progression. J Am Coll Surg. 2010 May;210(5):824-30, 831-2.

10. Brown CV, Zada G, Salim A, Inaba K, Kasotakis G, Hadjizacharia P, Demetriades D, Rhee P.

11. Indications for routine repeat head computed tomography (CT) stratified by severity of traumatic brain injury. $J$ Trauma. 2007 Jun;62(6):1339-44; discussion 1344-5.

12. Stein SC, Fabbri A, Servadei F. Routine serial computed tomographic scans in mild traumatic brain injury: when are they cost-effective? J Trauma. 2008 Jul;65(1):66-72.

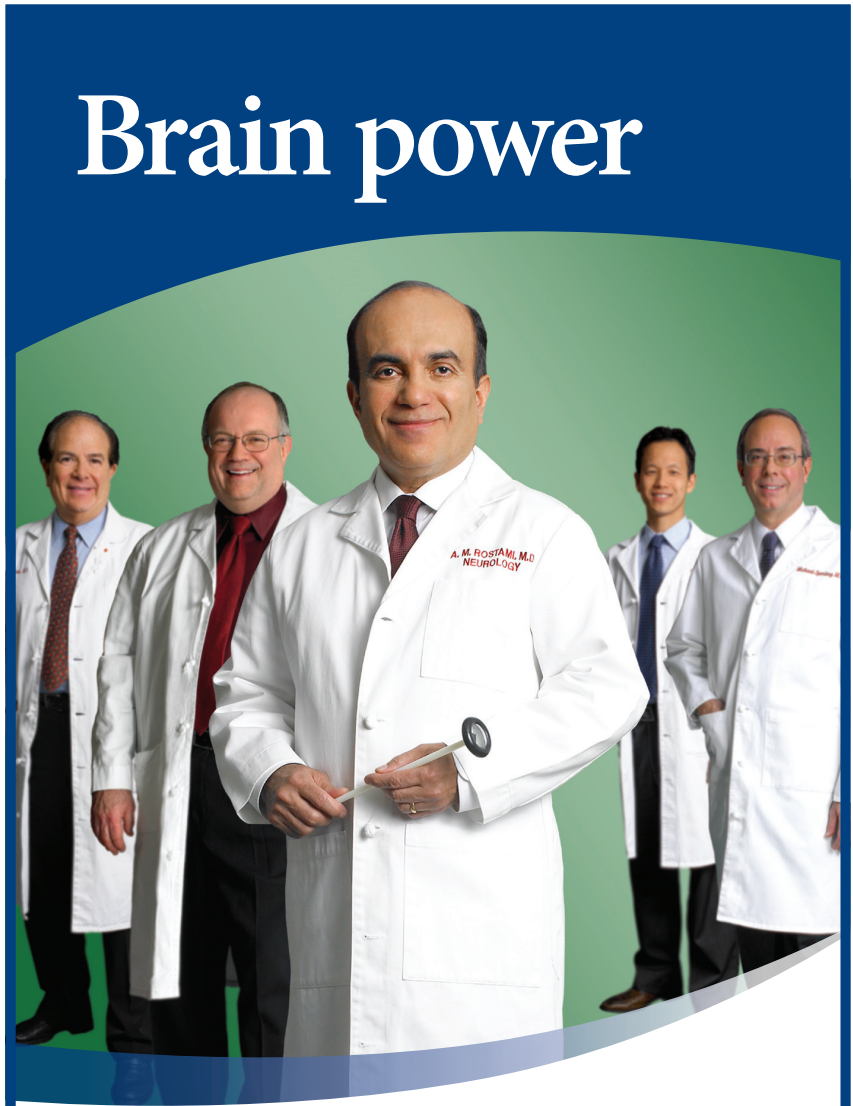

Jefferson neurologists have pioneered new treatments for stroke, and are on the leading edge in developing new treatments for everything from multiple sclerosis and Parkinson's disease to epilepsy and headache as well as other nerve and muscle diseases. Some are officers in prestigious national medical societies. Many are well-known in the worldwide medical community. Best of all, they work in our communityso they're available to see you.

The region's only hospital with an experienced, specialized staff dedicated to neuroscience.

Most insurance plans accepted, including Medicare.

\section{a. Jefferson.}

Hospital for Neuroscience

$$
\begin{aligned}
& \text { - Thomas Jefferson University Hospital } \\
& \text { - Jefferson Hospital for Neuroscience } \\
& \text { - Methodist Hospital }
\end{aligned}
$$

